\title{
BRIEF NEUTRINO PHYSICS UPDATE
}

\author{
J. W. F. VALLE \\ Instituto de Física Corpuscular, C.S.I.C. - Universitat de València \\ Edificio de Institutos de Paterna, Apartado 22085, E-46071 València, Spain
}

\begin{abstract}
The discovery of neutrino mass establishes the need for physics beyond the Standard Model. I summarize the status of two- and three-neutrino oscillation parameters from current solar, atmospheric, reactor and accelerator data. Future neutrinoless double beta decay experiments will probe the nature of neutrinos, as well as the absolute scale of neutrino mass, also tested by tritium beta decay spectra and cosmological observations. Sterile neutrinos do not provide a good way to account for the LSND hint, which needs further confirmation. Finally I sketch the main theoretical ideas for generating neutrino mass.
\end{abstract}

\section{Two-Neutrino Parameters}

In conjunction with the most recent SNO data with enhanced neutral current sensitivity (salt phase) 1 and the KamLAND reactor data 2 , solar neutrino experiments have now established the oscillation phenomenon. This closes the solar neutrino problem and opens an era of opportunity for learning more about the Sun 3 or about beyond-oscillations properties of neutrinos, such as magnetic moments 4 and non-standard interactions 567. Although wellmotivated by theory, such mechanisms can no longer account for the data and may only be present at a sub-leading level 8910. Similarly, the solid oscillation interpretation of the atmospheric neutrino data 11 leaves little room for beyond-oscillation non-standard physics 12 .

Neutrino masses 131415161718 have finally been discovered 19 . A complete analysis of recent solar, atmospheric, accelerator and reactor neutrino data has been given in Ref. $20 a$. This paper presents an updated determination of the neutrino oscillation parameters taking into account all data (see Ref. 20 for details) including the new solar neutrino data from the SNO-salt phase 1 . The resulting $90 \%, 95 \%, 99 \%$, and $3 \sigma 2$ d.o.f. C.L. regions in $\sin ^{2} \theta_{\text {SOL }}$, $\Delta m_{\mathrm{SOL}}^{2}$ allowed by all solar neutrino data before (lines) and after (shaded regions) the inclusion of the SNO-salt data are shown in Fig. 1 Also shown in this figure is $\Delta \chi^{2}$ as a function of $\sin ^{2} \theta_{\mathrm{SOL}}$ and $\Delta m_{\mathrm{SOL}}^{2}$, minimized with respect to the undisplayed parameter. One finds that especially the upper part of the

${ }^{a}$ See Refs. 2122 for extensive list of experimental solar and atmospheric neutrino references. For a discussion of other neutrino data analyses see Table 2 in 21 and the reviews in 19 .

dur03: submitted to World Scientific on November 10, 2018 


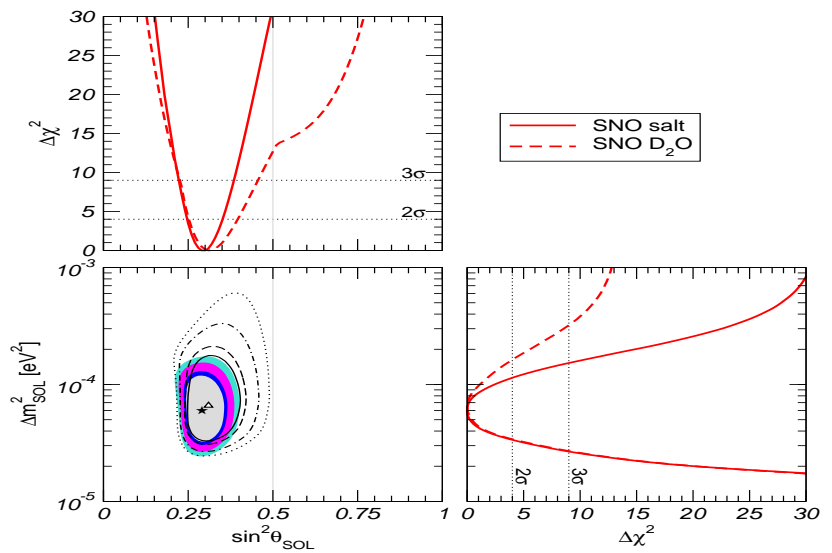

Figure 1. Two-neutrino solar neutrino oscillation parameters, from Ref. 20 .

LMA-MSW region and large mixing angles are strongly constrained by the new data, with $\sin ^{2} \theta_{\text {SOL }}=0.5$ excluded at more than $5 \sigma$. This rules out all bi-maximal models of neutrino mass 19 .

The first 145.1 days of KamLAND data have important implications on the determination of the solar neutrino parameters, as discussed, for example, in Ref. 21122]. Fig. 2 shows the projections of the allowed regions from all solar neutrino and KamLAND data at 90\%, 95\%, 99\%, and $3 \sigma$ C.L. for 2 d.o.f. onto the plane of $\sin ^{2} \theta_{\mathrm{SOL}}$ and $\Delta m_{\mathrm{SOL}}^{2}$ before (lines) and after (shaded regions) the inclusion of the SNO-salt data. Also shown is $\Delta \chi^{2}$ as a function of $\sin ^{2} \theta_{\text {SOL }}$ and $\Delta m_{\text {SOL }}^{2}$, minimized with respect to the undisplayed parameter. One sees that the SNO-salt results reject the previously allowed high-mass branch of $\Delta m_{\mathrm{SOL}}^{2}$ at about $3 \sigma$. Moreover, for the first time it is possible to obtain meaningful bounds on solar neutrino parameters at the $5 \sigma$ level, showing that neutrino physics has just entered the precision age.

Turning to the atmospheric neutrino parameters, we show in Fig. 3 the projection of the allowed regions from the global fit of all atmospheric data (details in Ref. 20, 23, onto the plane of the atmospheric neutrino parameters. The regions displayed correspond to $90 \%, 95 \%, 99 \%$, and $3 \sigma$ C.L. for 2 d.o.f. implied by the atmospheric+solar+CHOOZ data, while for the shaded regions also the $\mathrm{K} 2 \mathrm{~K}$ and KamLAND data are added. Also shown is the $\Delta \chi^{2}$ as a function of $\sin ^{2} \theta_{\text {ATM }}$ and $\Delta m_{\text {ATM }}^{2}$, minimized with respect to undisplayed parameters. One sees that the first 29 events from K2K 23 included here already constrain the upper region of $\Delta m_{\mathrm{ATM}}^{2}$. This should be contrasted with the lowering of $\Delta m_{\mathrm{ATM}}^{2}$ indicated by a recent preliminary reanalysis of 


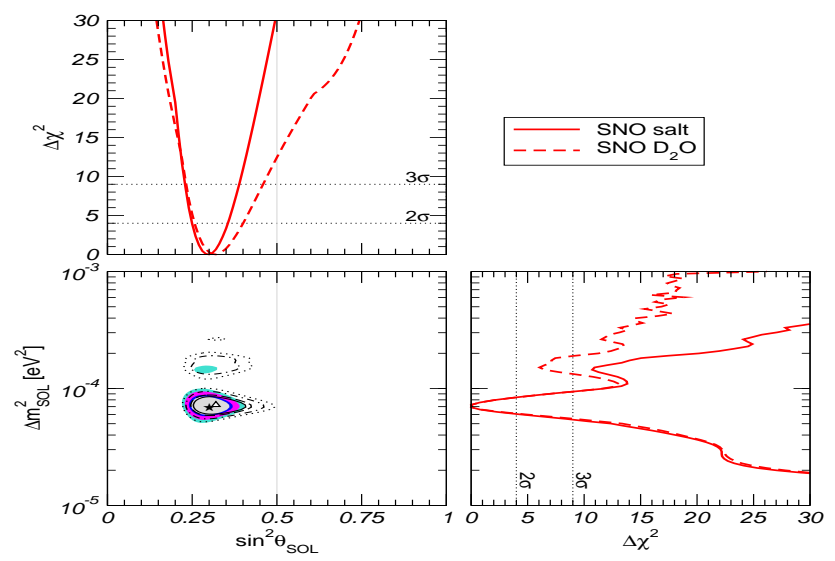

Figure 2. Two-neutrino solar+KamLAND neutrino oscillation parameters, from Ref. 20.

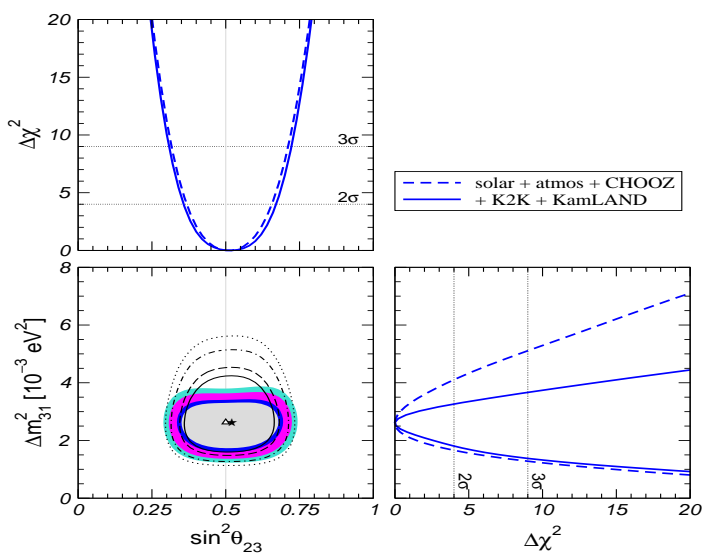

Figure 3. Two-neutrino atmospheric neutrino oscillation parameters from Ref. 20 .

the atmospheric data by the Super-Kamiokande collaboration presented at the Aachen EPS conference 24. While the two analyses differ, the value for $\Delta m_{\text {ATM }}^{2}$ quoted in 24 is statistically compatible with the result shown above. For $\Delta m_{\mathrm{ATM}}^{2}=2 \times 10^{-3} \mathrm{eV}^{2}$ and maximal mixing Ref. 20 obtains a $\Delta \chi^{2}=1.3$. 


\section{Three-Neutrino Parameters}

We now summarize the results of a global analysis combining all current solar, atmospheric, reactor and accelerator data in order to obtain the allowed three-neutrino oscillation parameters 20] The simpest three-neutrino lepton mixing matrix is parameterized as a product of three complex rotations $K=\omega_{12} \omega_{13} \omega_{23}, \omega_{i j}$ being a rotation in the $i j$ sector. This involves three mixing angles and three CP-violating phases 115 , one of which is the analogue of the quark $\mathrm{CP}$ phase, whose effect in oscillations we neglect, while the two Majorana phases 15 do not show up in oscillations but appear in lepton number violating processes 2526 . This way one is left with just the three angles in the neutrino oscillation analysis: $\theta_{12} \equiv \theta_{\text {SoL }}$ which governs solar neutrino oscillations, $\theta_{23} \equiv \theta_{\text {Атм }}$ which characterizes atmospheric neutrino oscillations, and $\theta_{13}$ which couples these two analyses.

$$
K=\left(\begin{array}{ccc}
c_{13} c_{12} & s_{12} c_{13} & s_{13} \\
-s_{12} c_{23}-s_{23} s_{13} c_{12} & c_{23} c_{12}-s_{23} s_{13} s_{12} & s_{23} c_{13} \\
s_{23} s_{12}-s_{13} c_{23} c_{12} & -s_{23} c_{12}-s_{13} s_{12} c_{23} & c_{23} c_{13}
\end{array}\right),
$$

Oscillations also involve the neutrino mass-squared differences $\Delta m_{\mathrm{SOL}}^{2} \equiv$ $\Delta m_{21}^{2} \equiv m_{2}^{2}-m_{1}^{2}$ and $\Delta m_{\mathrm{ATM}}^{2} \equiv \Delta m_{31}^{2} \equiv m_{3}^{2}-m_{1}^{2}$. Because of the hierarchy $\Delta m_{\mathrm{SOL}}^{2} \ll \Delta m_{\mathrm{ATM}}^{2}$ it is a good approximation to set $\Delta m_{\mathrm{SOL}}^{2}=0$ in the analysis of atmospheric and $\mathrm{K} 2 \mathrm{~K}$ data, and to set $\Delta m_{\mathrm{ATM}}^{2}$ to infinity for the analysis of solar and KamLAND data. The global fit to the data then involves five oscillation parameters $\sin ^{2} \theta_{12}, \sin ^{2} \theta_{23}, \sin ^{2} \theta_{13}, \Delta m_{21}^{2}, \Delta m_{31}^{2}$. The results of such three-neutrino analysis are summarized in Fig. 4. taken from Ref. 20, showing the allowed regions and $\chi^{2}$ projections for the above five oscillation parameters. The regions are at $90 \%, 95 \%, 99 \%$, and $3 \sigma$ C.L. for 2 d.o.f. for various parameter combinations. Also shown is $\Delta \chi^{2}$ as a function of the five oscillation parameters, minimized with respect to all undisplayed parameters. Finally, the best-fit values, $2 \sigma, 3 \sigma$ and $5 \sigma$ intervals ( 1 d.o.f.) for the three-flavour neutrino oscillation parameters derived from current solar, atmospheric, reactor (KamLAND and CHOOZ) and accelerator $(\mathrm{K} 2 \mathrm{~K})$ experiments are given in Table 1 of Ref. 20. It is remarkable that, for the first time, one can determine solar neutrino parameters at the $5 \sigma$ level, showing that neutrino physics has now entered the precision phase.

\section{Future agenda}

So far all CP phases are neglected in all current neutrino oscillation analyses. This is justified because the $\mathrm{CP}$ violating effects are suppressed 


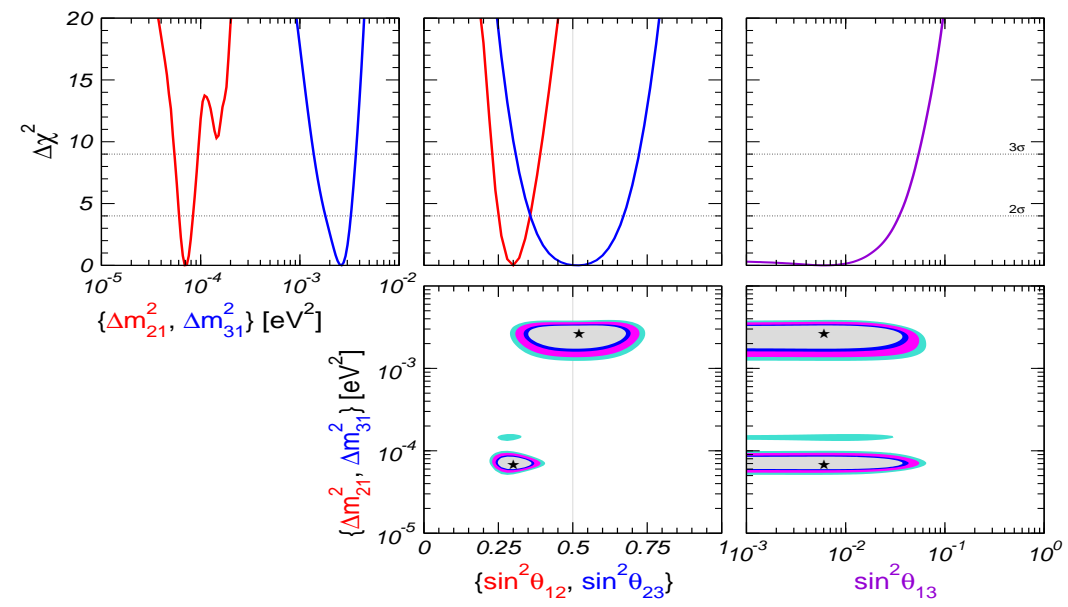

Figure 4. Three-neutrino oscillation parameters from Ref. 20].

due to the stringent limits on $\theta_{13}$ following mainly from reactor data 27 , shown in Fig. 5 On the left panel one can see the 90\%, 95\%, 99\%, and $3 \sigma$ allowed $\left(\sin ^{2} \theta_{13}, \Delta m_{\mathrm{ATM}}^{2}\right)$ regions from $\mathrm{CHOOZ}$ data alone (lines) and $\mathrm{CHOOZ}+$ solar+KamLAND data (shaded regions). Moreover leptonic CP violating effects are suppressed by the small mass splitting indicated by the solar neutrino data analysis. Indeed, in the 3 -neutrino limit, CP violation disappears as two neutrinos become degenerate 28 . Current data determine the ratio $\alpha \equiv \Delta m_{\mathrm{SOL}}^{2} / \Delta m_{\mathrm{ATM}}^{2}$ as shown in the right panel of Fig. 5
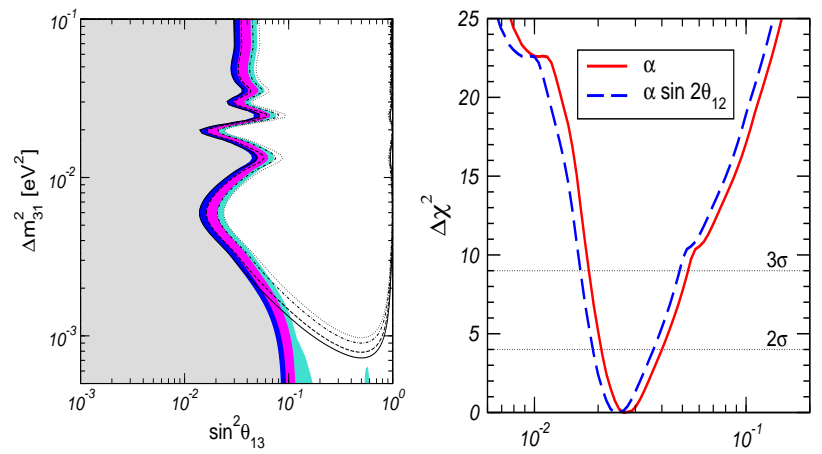

Figure 5. $\sin ^{2} \theta_{13}$ and $\alpha \equiv \Delta m_{\mathrm{SOL}}^{2} / \Delta m_{\mathrm{ATM}}^{2}$ from current neutrino data, from Ref. 20 
Now that the neutrino oscillation phenomenon has been confirmed, one may try to go a step further and test for the phenomenon of leptonic CP violation, either induced by the Dirac phase (oscillations) or by the Majorana phases 152625 through L-and-CP violating processes ${ }^{b}$. Let us start with oscillations. One sees that the value for $\alpha$ inferred from the global neutrino oscillation analysis and the reactor bound on $\sin ^{2} \theta_{13}$ both limit the prospects for probing $\mathrm{CP}$ violating effects at future neutrino oscillation experiments with superbeams or neutrino factories 30313233 . It will be challenge to probe such small effects, and this will require a near-detector in order to reject against the presence of non-standard neutrino interactions 34135 .

On the other hand, now that neutrino masses have been established, it is natural to check whether neutrinos are Majorana particles, as expected from theory 15 . Neutrinoless double beta decay 36 provides the most sensitive probe into the nature of neutrinos, irrespective of its theortetical origin 37 . There is indeed a new generation of proposed experiments aimed at detecting $\beta \beta_{0 \nu}$ with improved sensitivity $38 / 39$.

Although potentially sensitive to the Majorana $\mathrm{CP}$ phases present in the lepton mixing matrix ${ }^{15 \mid 25}$, current nuclear physics uncertainties still preclude a realistic way to test Majorana phases using this process 40, even if several isotopes are combined. As for other lepton number violating processes, these are strongly suppressed by the small masses of neutrinos and/or the V-A nature of the weak interaction. For example the L-violating neutrino oscillation probability involved in the "thought-experiment" proposed in Ref. 25$]$ is suppressed by $\left(m_{\nu} / E\right)^{2}$, while transition Majorana neutrino magnetic moments 4 also vanish in the massless neutrino limit 41 .

Let us now turn to another issue, namely, the number of light neutrinos. Are there more than three light neutrinos?

The LSND collaboration has claimed evidence for oscillations 42 which would strongly suggest the existence of a fourth (singlet) neutrino species at the electron-volt range, as could arise, say, due to some protecting global symmetry such as lepton number 4344445. However, a combined global four-neutrino study including also the solar, atmospheric and negative shortbaseline oscillation searches, such as Karmen, Bugey and CDHS, strongly prefer the minimal three light-neutrino hypothesis 214647]. The data rule out the possibility of symmetric $(2+2)$ schemes, because in this case sterile neutrinos take part in both solar and atmospheric oscillations. Though strongly disfavoured by short-baseline experiments, the presence of a light

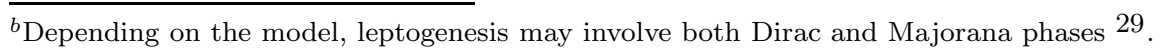

dur03: submitted to World Scientific on November 10, 2018 
sterile neutrino in a $(3+1)$ scheme may still be allowed, since it can be chosen to decouple from solar and atmospheric oscillations. Data from cosmology, including CMB data from WMAP 48 4950/51 and the 2dFGRS large scale structure surveys ${ }^{[52}$ lead to further restrictions, especially on large $\Delta m_{\mathrm{LSND}}^{2}$ values.

\section{Neutrino Theory: Top-Down versus Bottom-Up}

The theoretical setting involved in the description of current neutrino oscillation experiments was laid out long ago 15 , including the two-component quantum description of massive Majorana neutrinos and the gauge theoretic characterization of the lepton mixing matrix. The other crucial ingredient was the formulation of neutrino oscillations in the presence of matter 56 .

The origin of neutrino mass remains as much of a mystery today as it was back in the eighties. Much of the early theoretical effort was motivated in part by the idea of unification which introduced the seesaw mechanism 13114 . Although first formulated in the context of the $S O(10)$ group, it was soon realized that the seesaw idea can be applied to left-right symmetric theories 16 , or the simplest effective Standard Model gauge framework 1511718. While the $S O(10)$ or $S U(2)_{L} \otimes S U(2)_{R} \otimes U(1)$ seesaw formulations have the virtue of relating the small neutrino mass to the dynamics of parity (gauged B-L) violation, the effective $S U(2) \otimes U(1)$ description is more general and applies to any theory, for example with ungauged B-L 1718. It is also worth-noting that the general seesaw scheme implies a Higgs triplet contribution to neutrino masses, from an induced tadpole or an elementary scalar vacuum expectation value 151653 .

However, it is worth stressing that the seesaw is just one way of generating the fundamental dimension-five neutrino mass operator 54. Such may also arise from physics "just around the corner". One example is provided by certain super-string-inspired models 55 ]. Indeed in such "anti-seesaw" models neutrino masses vanish as the B-L scale goes to zero, rather than infinity.

An alternative origin for neutrino mass is provided by the idea of low energy supersymmetry 565758 in schemes that break R parity through a sneutrino vacuum expectation value 5960 . These lead effectively to bilinear $\mathrm{R}$ parity violation 61. The novelty here is that neutrino mixing angles can be tested at accelerator experiments 626364. Hybrid alternatives involving triplet Higgs bosons and supersymmetry are possible 65 .

In summary there is no "road-map" for the ultimate theory of neutrino

dur03: submitted to World Scientific on November 10, 2018 
mass, a wide variety of pathways remains open. In this context one expects small residual effects associated to non-standard weak interaction properties of neutrinos. These may follow from the particular structure of the charged and neutral currents expected in theories where neutrino masses follow from the existence of isosinglet leptons $\underline{15}$ or from alternative low energy radiative mechanisms for neutrino mass generation $[66$ and their variants.

This work was supported by Spanish grant BFM2002-00345, European Commission RTN grant HPRN-CT-2000-00148, European Science Foundation Neutrino Astrophysics Network and a Humboldt Research Award. This written version includes the SNO-salt data, analysed in detail in Ref. 20.

\section{References}

1. S. N. Ahmed et al. nucl-ex/0309004

2. K. Eguchi et al. Phys. Rev. Lett., 90:021802, 2003.

3. C. Burgess et al. Astrophys. J., 588:L65, 2003, and astro-ph/0304462

4. J. Schechter and J. W. F. Valle. Phys. Rev., D24:1883, 1981. Err. Phys. Rev. D25, 283 (1982). E. Kh. Akhmedov. Phys. Lett., B213:64-68, 1988. C. S. Lim and W. J. Marciano. Phys. Rev., D37:1368, 1988.

5. L. Wolfenstein. Phys. Rev., D17:2369, 1978.

6. S. P. Mikheev \& A. Yu. Smirnov. Sov. J. Nucl. Phys., 42:913-917, 1985.

7. J. W. F. Valle. Phys. Lett., B199:432, 1987.

8. J. Barranco, et al Phys. Rev., D66:093009, 2002, hep-ph/0207326 (v3).

9. M. Guzzo et al. Nucl. Phys., B629:479-490, 2002, hep-ph/0112310(v3)

10. W. Grimus, et al, Nucl. Phys., B648:376-396, 2003, hep-ph/0208132 (v2)

11. Y. Fukuda et al. Phys. Rev. Lett., 81:1562-1567, 1998.

12. N. Fornengo et al, Phys. Rev., D65:013010, 2002, hep-ph/0108043 (v3).

13. M. Gell-Mann, P. Ramond, and R. Slansky. Print-80-0576 (CERN).

14. T. Yanagida. KEK lectures. ed. Sawada and Sugamoto (KEK, 1979).

15. J. Schechter and J. W. F. Valle. Phys. Rev., D22:2227, 1980.

16. R. N. Mohapatra and G. Senjanovic. Phys. Rev., D23:165, 1981.

17. Y. Chikashige, R. Mohapatra \& R. Peccei Phys. Lett., B98:265, 1981.

18. J. Schechter and J. W. F. Valle. Phys. Rev., D25:774, 1982.

19. S. Pakvasa and J. W. F. Valle. Proceedings of the Indian National Academy of Sciences on Neutrinos, hep-ph 0301061 V. Barger, D. Marfatia, and K. Whisnant. hep-ph/0308123 S. Bilenky, C. Giunti \& W. Grimus. Eur. Phys. J., C1:247-253, 1998.

20. M. Maltoni et al, hep-ph/0309130

21. M. Maltoni et al, Phys. Rev., D67:013011, 2003, hep-ph/0207227 (v3) 
22. M. Maltoni, T. Schwetz \& J. W. F. Valle. Phys. Rev., D67:093003, 2003.

23. M. H. Ahn et al. Phys. Rev. Lett., 90:041801, 2003.

24. Y. Hayato, talk at the HEP2003 conference, Aachen, Germany, 2003.

25. J. Schechter and J. W. F. Valle. Phys. Rev., D23:1666, 1981.

26. M. Doi et al, Phys. Lett., B102:323, 1981.

27. M. Apollonio et al. Phys. Lett., B466:415-430, 1999.

28. J. Schechter and J. W. F. Valle. Phys. Rev., D21:309, 1980.

29. M. Fukugita and T. Yanagida. Phys. Lett., B174:45, 1986.

30. M. Apollonio et al. hep-ph/0210192.

31. M. Freund, P. Huber, and M. Lindner. Nucl. Phys., B615:331-357, 2001.

32. C. Albright et al. hep-ex/0008064

33. A. Cervera et al. Nucl. Phys., B579:17-55, 2000.

34. P. Huber, T. Schwetz \& J. W. Valle. Phys. Rev. Lett., 88:101804, 2002.

35. P. Huber, T. Schwetz \& J. W. F. Valle. Phys. Rev., D66:013006, 2002.

36. Lincoln Wolfenstein. Phys. Lett., B107:77, 1981.

37. J. Schechter and J. W. F. Valle. Phys. Rev., D25:2951, 1982.

38. S. R. Elliott \& P. Vogel. Ann. Rev. Nucl. Part. Sci., 52:115-151, 2002.

39. H. V. Klapdor-Kleingrothaus et al. hep-ph/9910205

40. V. Barger et al, Phys. Lett., B540:247-251, 2002.

41. P. B. Pal and L. Wolfenstein. Phys. Rev., D25:766, 1982.

42. A. Aguilar et al. Phys. Rev., D64:112007, 2001.

43. J. T. Peltoniemi and J. W. F. Valle. Nucl. Phys., B406:409, 1993. J. T. Peltoniemi, D. Tommasini \& J. W. F. Valle. Phys. Lett., B298:383, 1993.

44. D. O. Caldwell and R. N. Mohapatra. Phys. Rev., D48:3259-3263, 1993.

45. C. Giunti \& M. Laveder. http://www.to.infn.it/ giunti/NU/.

46. M. Maltoni et al, Nucl. Phys., B643:321-338, 2002.

47. M. Maltoni et al, hep-ph/0305312

48. D. N. Spergel et al. astro-ph/0302209

49. P. Crotty, J. Lesgourgues, and S. Pastor. Phys. Rev., D67:123005, 2003.

50. O. Elgaroy and O. Lahav. JCAP, 0304:004, 2003.

51. Steen Hannestad. astro-ph/0303076

52. Max Tegmark et al, Mon. Not. Roy. Astron. Soc., 335:887, 2002.

53. T. P. Cheng and Ling-Fong Li. Phys. Rev., D22:2860, 1980.

54. S. Weinberg. Harvard preprint - HUTP-80-A038

55. R. N. Mohapatra and J. W. F. Valle. Phys. Rev., D34:1642, 1986.

56. M. A. Diaz et al, hep-ph/0302021 Phys.Rev., D68:013009, 2003

57. M. Hirsch et al, Phys. Rev., D62:113008, 2000. Err. D65:119901,2002.

58. J. C. Romao et al, Phys. Rev., D61:071703, 2000.

59. G. G. Ross \& J. W. F. Valle Phys. Lett., B151:375, 1985; J. R. Ellis et al, Phys. Lett., B150:142, 1985. 
60. A. Masiero and J. W. F. Valle. Phys. Lett., B251:273-278, 1990.

61. M. Diaz, J. C. Romao \& J. W. F. Valle. Nucl. Phys., B524:23-40, 1998.

62. M. Hirsch et al, Phys. Rev., D66:095006, 2002.

63. W. Porod et al, Phys. Rev., D63:115004, 2001.

64. D. Restrepo, W. Porod \& J. W. F. Valle. Phys. Rev., D64:055011, 2001.

65. D. Aristizabal et al, Phys.Rev., D68: 033006, 2003

66. K. Babu Phys. Lett. B203:132, 1988; A. Zee Phys. Lett. B93:389, 1980. 